\title{
Investigation of mid-IR picosecond image upconversion
}

\author{
Mathez, Morgan David; Pedersen, Christian; Rodrigo, Peter John; Tidemand-Lichtenberg, Peter
}

\section{Published in:}

Proceedings of SPIE

Link to article, DOI:

$10.1117 / 12.2251107$

Publication date:

2017

Document Version

Publisher's PDF, also known as Version of record

Link back to DTU Orbit

Citation (APA):

Mathez, M. D., Pedersen, C., Rodrigo, P. J., \& Tidemand-Lichtenberg, P. (2017). Investigation of mid-IR picosecond image upconversion. In Proceedings of SPIE (Vol. 10088). [100880L] SPIE - International Society for Optical Engineering. Proceedings of SPIE - The International Society for Optical Engineering https://doi.org/10.1117/12.2251107

\section{General rights}

Copyright and moral rights for the publications made accessible in the public portal are retained by the authors and/or other copyright owners and it is a condition of accessing publications that users recognise and abide by the legal requirements associated with these rights.

- Users may download and print one copy of any publication from the public portal for the purpose of private study or research.

- You may not further distribute the material or use it for any profit-making activity or commercial gain

- You may freely distribute the URL identifying the publication in the public portal 


\title{
Investigation of Mid-IR picosecond image upconversion
}

\author{
Morgan Mathez, Christian Pedersen, Peter John Rodrigo and Peter Tidemand-Lichtenberg \\ DTU Fotonik, Technical University of Denmark, 4000 Roskilde, Denmark
}

\begin{abstract}
Imaging and spectroscopy in the mid-infrared (Mid-IR) wavelength region have received considerable attention in recent years. The reason is the high Mid-IR spectral specificity of many gases and complex molecules. In this pilot study we focus on picosecond upconversion imaging exploiting the $\chi^{(2)}$ nonlinearity of a bulk lithium niobate crystal as a means to convert the optical Mid-IR signal into the visible wavelength region, thus allowing the use of fast and sensitive silicon based CCD cameras. The picosecond upconversion system is synchronously pumped in order to increase the quantum efficiency, hence allowing for upconversion of faint pulsed Mid-IR light.
\end{abstract}

Keywords: Nonlinear wave mixing, Infrared imaging, Upconversion, pulsed laser

\section{INTRODUCTION}

Upconversion of infrared radiation into the visible spectrum is an emerging technology showing good promise as an alternative to conventional infrared cameras, which often require cooling and suffer from limited sensitivity and spectral response. ${ }^{1}$ In contrast, upconverted mid-infrared (Mid-IR) radiation can be detected by means of cost effective, highly sensitive visible to near infrared (Near-IR) detectors and cameras, thus making upconversion an attractive technique for Mid-IR detection and imaging. Upconversion has been applied in a diverse fields such as astrophysics to observe thermal radiation from stars, ${ }^{2}$ to detect natural gas leakage and the distribution of the greenhouse gases, ${ }^{3}$ and to convert image information into the visible region using Mid-IR supercontinuum light sources. ${ }^{4}$ Furthermore, using infrared sources with short pulse width, it becomes possible to study phenomenon such as the intraband relaxation dynamics of particles ${ }^{5,6}$ and the relaxation of molecule stretch vibrations. ${ }^{7}$

In this paper we discuss the experimental results of upconversion imaging using short Mid-IR pulses (1 ps). The upconversion takes place in a simple, single pass configuration inside a bulk nonlinear (lithium niobate) crystal. Different aspects are analyzed, such as the intensity and spectral distribution of the upconverted radiation. Finally, we experimentally compare the performance of the upconversion imaging system using two different signal spectral bandwidths and note the effects on field-of-view and spatial resolution.

\section{PRINCIPLE OF UPCONVERSION IMAGING}

The purpose of this work is to experimentally investigate the upconversion of a Mid-IR beam with spatial information in to the visible light wavelength range. Upconversion relies on sum frequency generation (SFG). The SFG or upconversion process involves mixing two optical beams to produce a third with a shorter wavelength. Two physical quantities need to be conserved. The first one is conservation of energy in the SFG process which needs to be obeyed strictly. The second quantity relates to the conservation of the vectorial momentum. The vector sum of the two input wave vectors should match the wave vector of the upconverted beam in order to reach the highest conversion efficiency. It is simple to understand this by representing these three vectors in the $\mathrm{k}$ vector space. Figure 1a shows the entire locus of the three $\mathrm{k}$ vectors involved. Considering a type I upconversion scheme, the incoming Mid-IR signal and the pump are ordinary polarized, thus their refractive indexes do not depend on the propagation angle inside the nonlinear crystal. However the upconverted beam is extraordinary polarized, therefore its refractive index can be tuned by rotating the nonlinear crystal angle $\left(\theta_{c}\right)$. This mechanism can then be used to obtain phase matching, i.e. conservation of momentum. In contrast to the need for exact conservation of energy, the conservation of momentum need not, but will then result in lower conversion efficiency.

Further author information:

Morgan Mathez: E-mail: modam@fotonik.dtu.dk

Nonlinear Frequency Generation and Conversion: Materials and Devices XVI, edited by
Konstantin L. Vodopyanov, Kenneth L. Schepler, Proc. of SPIE Vol. 10088, 100880L

(c) 2017 SPIE · CCC code: 0277-786X/17/\$18 - doi: 10.1117/12.2251107

Proc. of SPIE Vol. 10088 100880L-1 


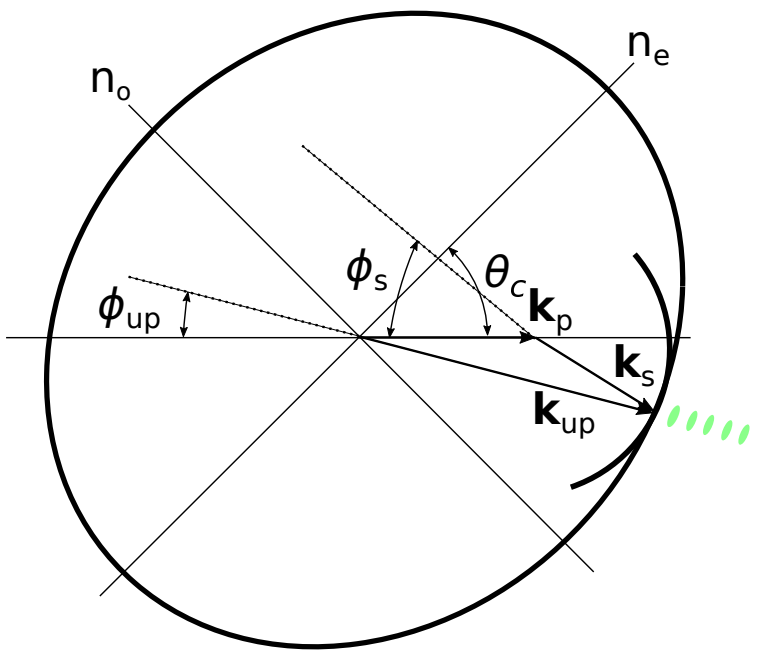

(a)

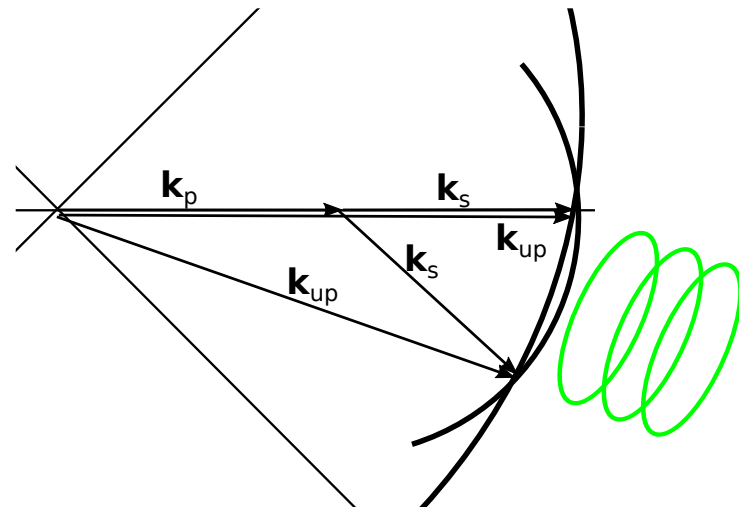

(b)

Figure 1: Phase matching in the k space for (a) a single phase matching solution and (b) a collinear phase matching (as a part of multiple solutions).

A single phase matched solution can be achieved with the correct angle $\theta_{c}$. In this special case the locus of $\mathbf{k}_{s}$ and $\mathbf{k}_{u p}$ intersect in one point only, producing a single point in the image plane (see Fig. 1a). This special case represents a noncollinear phase matched solution, since generally $\mathbf{k}_{s}, \mathbf{k}_{p}$ and $\mathbf{k}_{u p}$ are not parallel. In order to achieve collinear phase matching, the crystal angle $\left(\theta_{c}\right)$ has to be tuned until $\mathbf{k}_{s}, \mathbf{k}_{p}$ and $\mathbf{k}_{u p}$ are parallel. The locus of $\mathbf{k}_{s}$ then generally intersects the locus of $\mathbf{k}_{u p}$ at two angles. Moreover the locus of $\mathbf{k}_{u p}$, which is an ellipsoid in 3D, has an eccentricity close to 0 . Therefore the shape of the intersection in 3D of these two loci can be approximated by a circle. The radius of the circle increases with $\phi_{s}$ with the single solution in Fig. 1a representing a minimum. Figure $1 \mathrm{~b}$ shows the connection between $\phi_{s}$ and the upconverted signal beam cone. The upconverted cone of light is re-imaged using a $2 \mathrm{f}$ set-up $(\mathrm{f}=150 \mathrm{~mm})$ to form a ring in the image plane (See Fig .2). Likewise a $2 \mathrm{f}$ set-up $(\mathrm{f}=40 \mathrm{~mm}$ ) is used to transform light from the object plane into proportional angles passing the the nonlinear crystal. The resulting $4 \mathrm{f}$ set-up forms the basis of the imaging process. A large signal beam diameter is able to illuminate a large object, however at the cost of a lower intensity compared to a small beam diameter.

\section{EXPERIMENTAL SETUP}

The angle $\phi_{u p}$ corresponding to the single phase matched solution is slightly different from the collinear alignment. This difference needs to be corrected for when designing the actual setup in order to produce a full circular rather than a crescent shape intensity distribution. Figure 2 illustrates this aspect of the alignment requirement. In Fig. 2 it is the Mid-IR signal beam (in orange) that is changed from the collinear case. We note that in some cases it could be more convenient to change the angle of the pump beam, which would produce the same effect.

The present experiment uses a $1877 \mathrm{~nm}$ Mid-IR signal beam with two possible options for the bandwidth, 11 and $150 \mathrm{~nm}$ respectively defined as the full width at half maximum (FWHM) of the spectrum, a power of $5 \mathrm{~mW}$ and $130 \mathrm{~mW}$ respectively. The pump beam has a center wavelength of $1550 \mathrm{~nm}$, a bandwidth of $1 \mathrm{~nm}$ after a band pass filter and an average power of $43 \mathrm{~mW}$. Both beams are synchronously pulsed with a pulse width of $1 \mathrm{ps}$, and a repetition rate of $21.8 \mathrm{MHz}$. Since 1 ps represents a spatial extent of only $0.3 \mathrm{~mm}$ in free space, temporal synchronization has to be accurate in order to ensure that the pulses from the Mid-IR signal beam overlap the pulses from the pump beam inside the bulk nonlinear crystal (lithium niobate). This has been accomplished using a variable path delay. A retro-reflector has been mounted on a translation stage to perform a fine temporal alignment of the pulses, thus increasing the upconversion efficiency.

The object is illuminated with a Gaussian beam having a beam waist diameter of $19.5 \mathrm{~mm}$. The transmitted beam is collected using a lens with a focal lens of $40 \mathrm{~mm}$, producing a Fourier transformed image of the object 


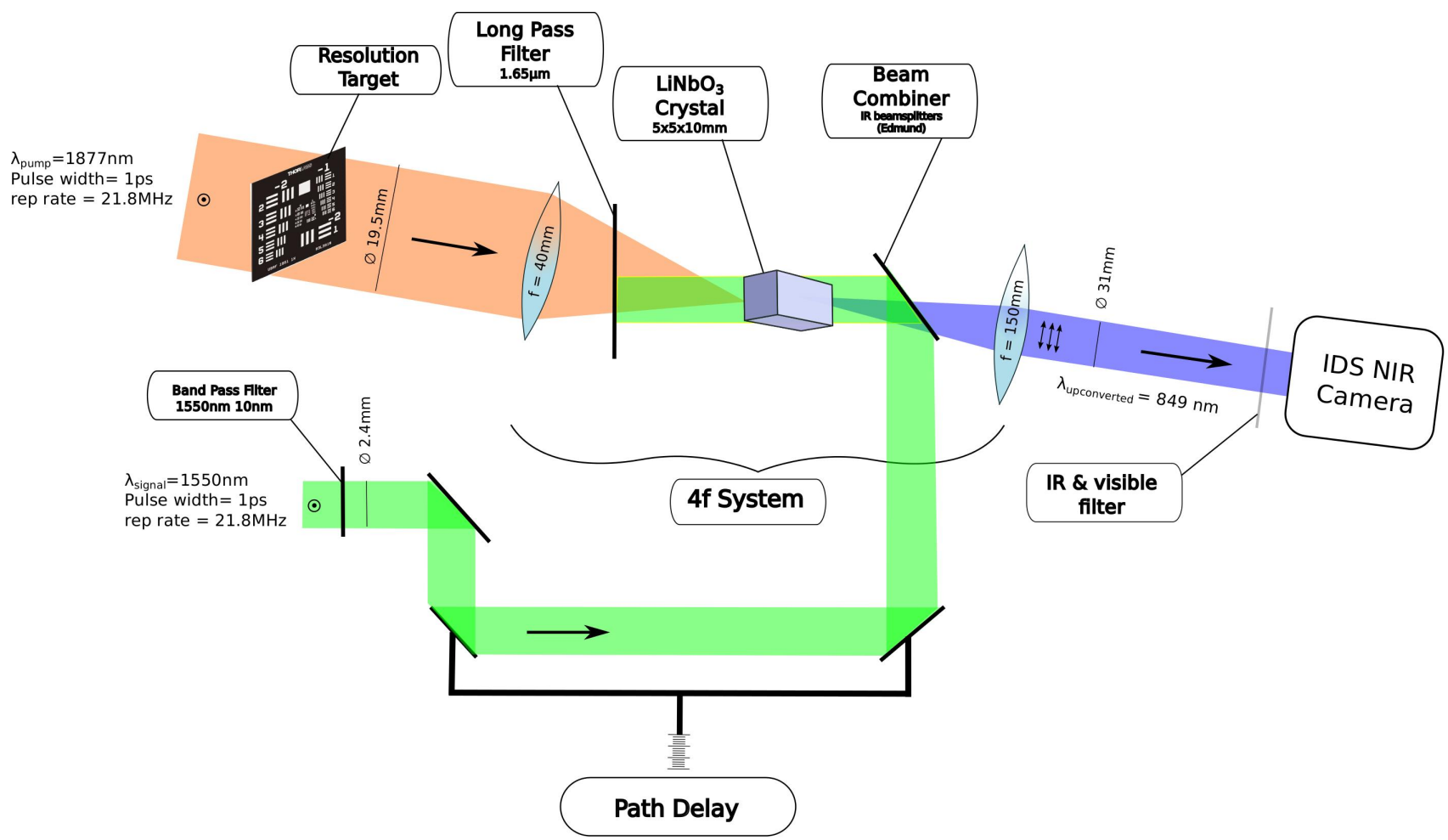

Figure 2: Diagram of the experimental setup. The Mid-IR and the pump beams are depicted in orange and green respectively and are both vertically polarized. The upconverted beam is shown in green and is horizontally polarized.

at the back focal plane. The nonlinear crystal is positioned in the mentioned Fourier plane, where also the upconversion takes place. The lens $(\mathrm{f}=40 \mathrm{~mm}$ ) has been chosen with the smallest possible focal length (according to the available space) in order to generate the smallest transverse spatial frequency distribution of the object in the Fourier plane, thus maximizing the spatial resolution with the actual pump beam waist diameter $(2.4 \mathrm{~mm})$. The limited space between the lens, $\mathrm{f}=40 \mathrm{~mm}$ and the nonlinear crystal force us to position the beam combiner after the nonlinear crystal, and reflect the pump beam back in to the crystal using a long pass filter as a mirror (See Fig. 2). The upconverted image $(849 \mathrm{~nm}$ ) is reconstructed with a Fourier transforming lens, $\mathrm{f}=150 \mathrm{~mm}$, and acquired by a $6.8 \mathrm{~mm} \times 5.4 \mathrm{~mm}$ silicon CCD camera chip (IDS; UI-3240CP-NIR-GL).

\section{INTENSITY AND SPECTRAL DISTRIBUTION}

The present section discusses the ring pattern formation in the image plane. It has been mentioned previously, that the full circle distribution of the intensity can be realized by tilting one of the two input beams. However the polychromatic nature of the two sources has not been investigated yet. In the following the pump beam is considered monochromatic in comparison with the Mid-IR signal spectrum. In this study two different MidIR signal beam spectrum are used, having a $11 \mathrm{~nm}$ and $150 \mathrm{~nm}$ bandwidth respectively. The different signal wavelengths affect at the same time the length and the direction of $\mathbf{k}_{s}$ and $\mathbf{k}_{u p}$, in which phase matching occurs. A polychromatic beam will consequently generate different upconverted ring structures in the image plane. It is of interest to investigate the distribution of these rings.

Two cases can be studied; one for each bandwidth. Figure 3 depicts the the upconverted beam intensity distribution for both cases. The $11 \mathrm{~nm}$ bandwidth produces a thin symmetric ring compared to its radius (see Fig. 3a), since the highest and lowest wavelength are close to each other compared to the central wavelength. However a significant thickness can be observed when the bandwidth increases to $150 \mathrm{~nm}$. Even more interesting is the asymmetry of the distribution. This effect is not associated with the misalignment of the Mid-IR signal but is rather the result of an asymmetry in the the phase matching solutions. Since the main axis of the ellipsoid in Fig. 1a is in the same plane as for the signal and the pump beam, this phase matching asymmetry occurs 

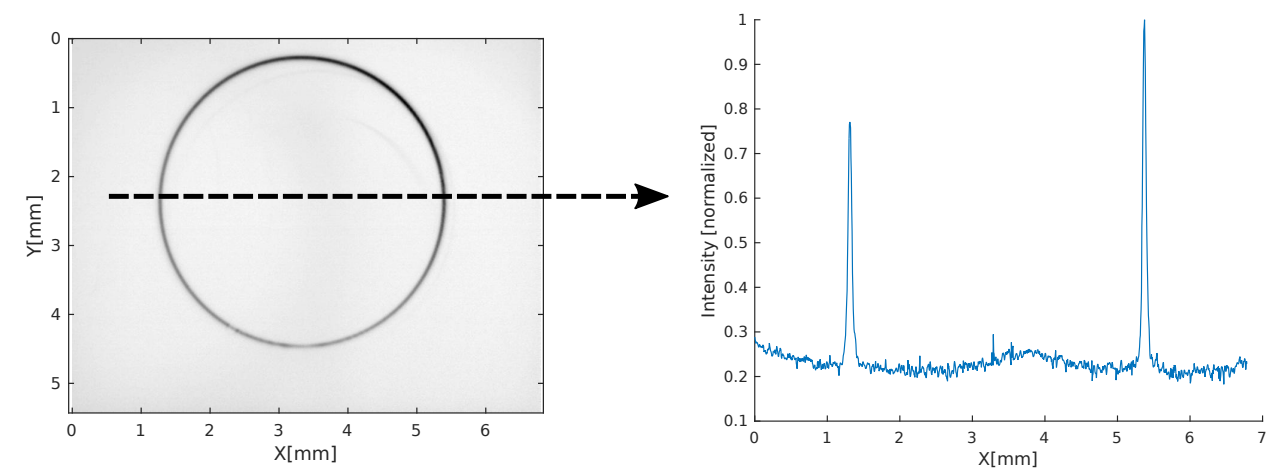

(a)
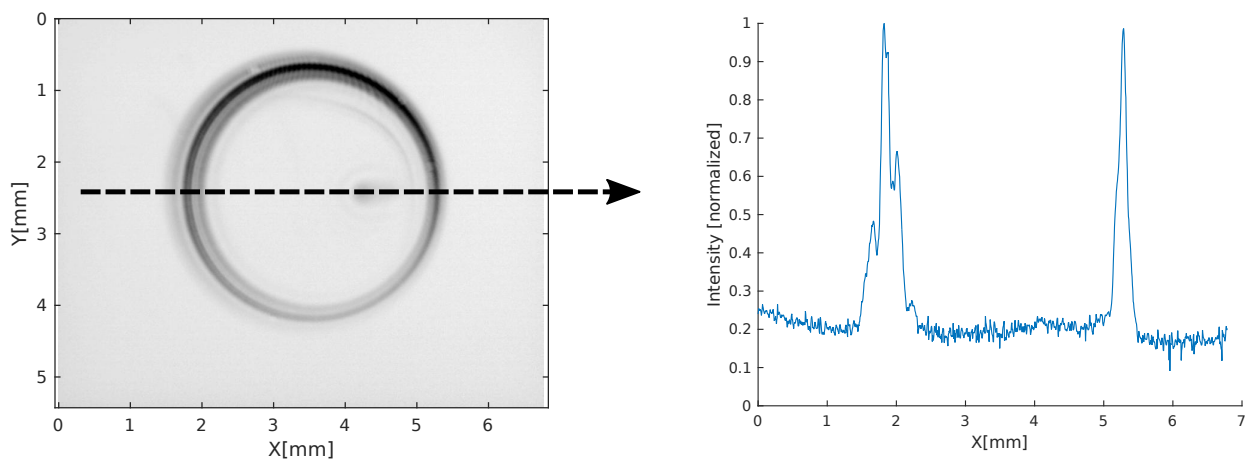

(b)

Figure 3: The upconverted beam intensity distribution for two different signal beam spectra. The bandwidth is (a) 11 $\mathrm{nm}$ and (b) $150 \mathrm{~nm}$.

only in this plane. Figure 3 illustrates this feature; the distribution of the phase matching solution is symmetric around the $\mathrm{Y}$ axis only. An analysis of the upconverted beam spectral distribution (measured by a spectrometer), produced by the $150 \mathrm{~nm}$ bandwidth has been performed to illustrate this asymmetry.

The first information provided by Fig. 4 is a confirmation of the asymmetry in the spectral domain. Moreover each side of the ring has the same bandwidth, meaning that the same Mid-IR signal bandwidth is upconverted at different positions of the circle. It also proves that the actual asymmetry in the intensity distribution does not come from an asymmetry in the signal bandwidth. Going one step further the bandwidth of the Mid-IR signal can be analyzed from the actual bandwidth of the upconverted beam. From Fig. 4 it can be seen that the upconverted bandwidth is $30 \mathrm{~nm}$. Since the pump beam at $1550 \mathrm{~nm}$ is almost monochromatic, it possible to calculate the bandwidth of the signal beam by applying the conservation of energy. Doing so gives a signal beam bandwidth of $150 \mathrm{~nm}$. This result matches perfectly with the actual spectrum of the signal beam measured directly with a Mid-IR spectrometer.

\section{EXPERIMENTS ON SPATIAL RESOLUTION}

The imaging properties of upconversion are studied in this section. The main purpose is to investigate some parameters which affect the resolution of the image. The 8 lines $/ \mathrm{mm}$ pattern of the resolution target is selected for this experiment in order to characterize different imaging aspects. 


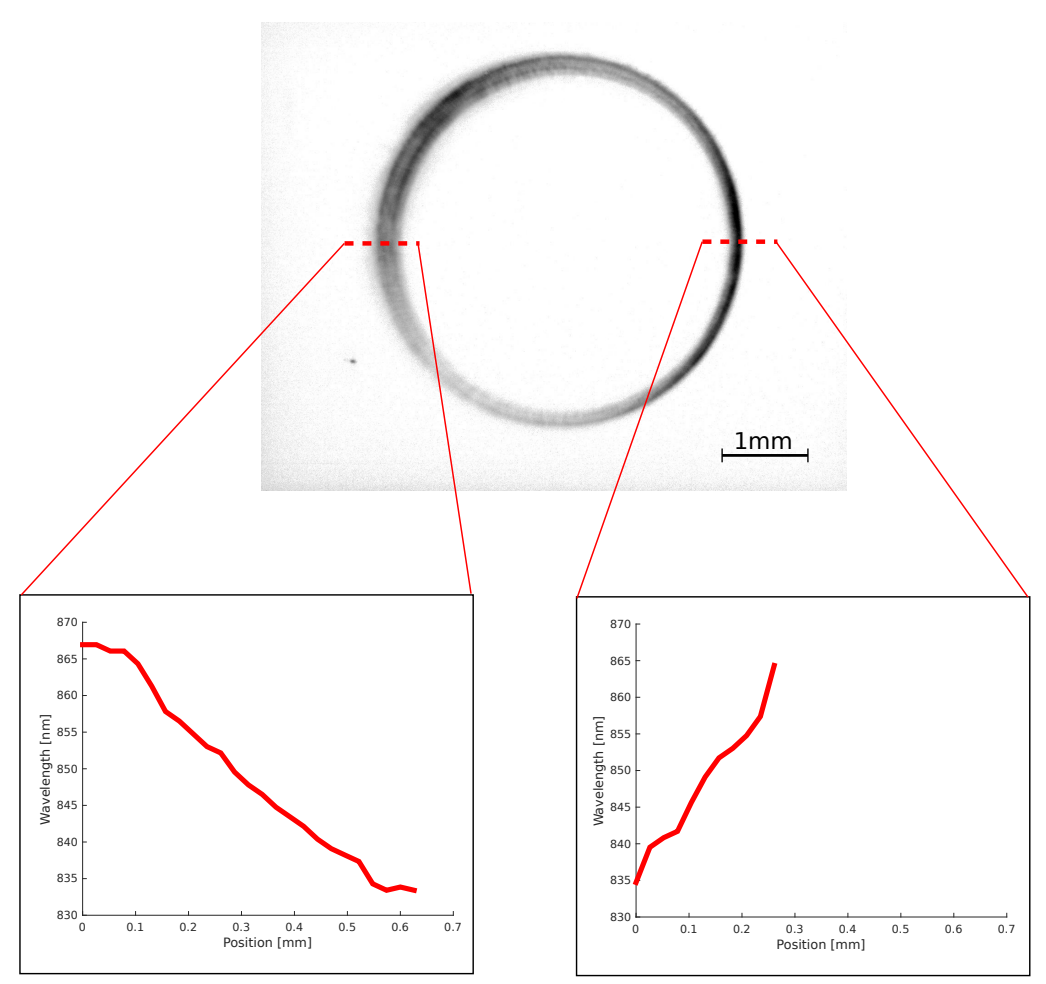

(a)

(b)

Figure 4: Linear scan of the spectrum distribution of the upconverted beam measured by a spectrometer.

\subsection{Influence of Mid-IR spectral bandwidth}

Since we have two possible options for the spectral width of the Mid-IR signal, it is possible to experimentally demonstrate the influence of the bandwidth on the spatial resolution. The object is thus illuminated successively by the $11 \mathrm{~nm}$ and $150 \mathrm{~nm}$ bandwidth signal beam respectively. The results are shown in Fig. 5 .

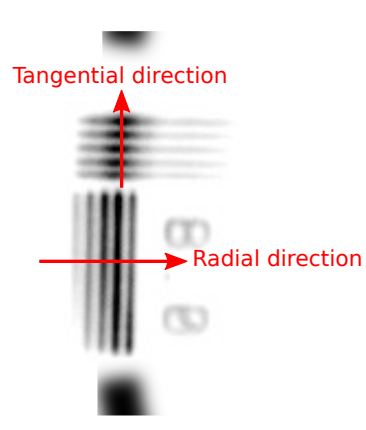

(a)

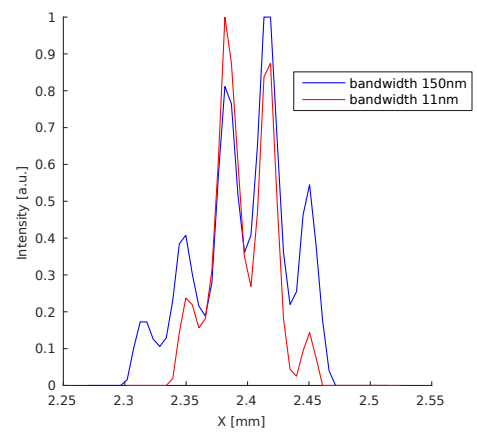

(b)

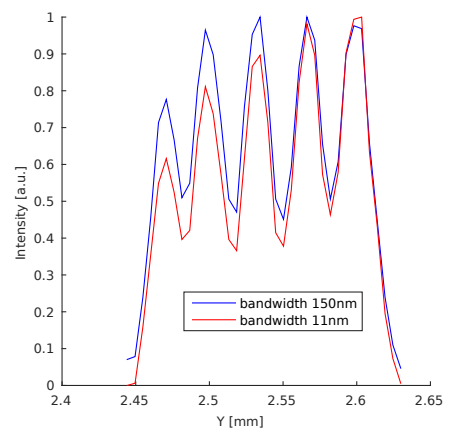

(c)

Figure 5: (a) Image of the 8 lines/mm area of a resolution target. Linear intensity scan in the (b) radial and (c) tangential direction

Figure 5 a represents the upconversion of the 8 lines/mm pattern of the resolution target when probed by the signal beam. Two orthogonal directions are scanned. The radial direction highlights a major difference between the two choices of bandwidths. The $150 \mathrm{~nm}$ bandwidth is able to illuminate a larger zone of the object in the radial direction. This is expected as discussed in the previous sections; the larger the bandwidth the thicker the ring or effective field-of-view. Moreover the resolution does not vary for the same intensity level (see the two central peaks in Fig. 5c). This observation is even more obvious for the scan along the tangential direction, since 
both bandwidths produce the same image. The conclusion is that the polychromatic nature of the signal beam influences the field-of-view but does not seem to produce any major blurring effect.

\subsection{Image resolution vs object position}

A central question for aberration for image formation is whether the point spread function is independent of the object position $(\mathrm{x}, \mathrm{y})$ or not. As discussed previously the intensity distribution has circular symmetry, thus lending itself to a description in polar coordinates. First the angular dependency is investigated. Therefore we examine the spatial resolution at four different positions of a particular ring (see Fig. 6a).

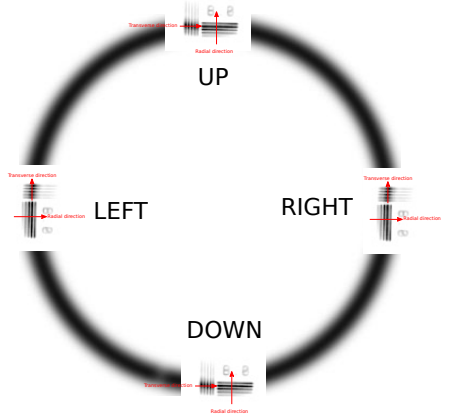

(a)

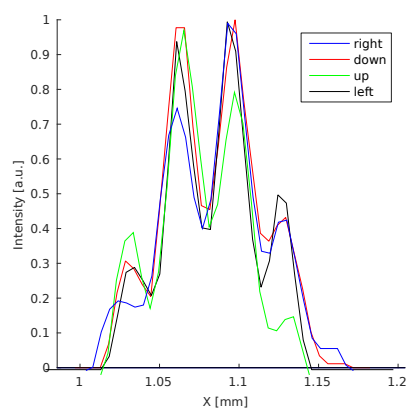

(b)

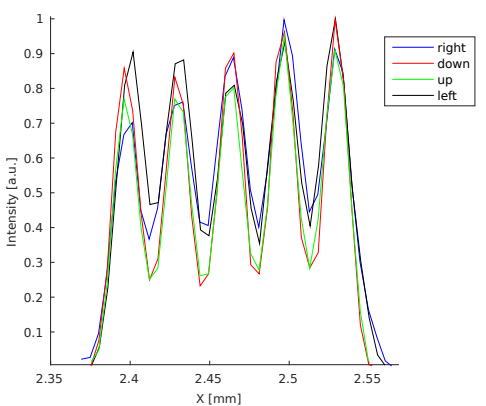

(c)

Figure 6: (a) The 8 lines/mm area is positioned in the four quadrants successively. Linear intensity scan in the (b) radial and (c) tangential direction. The dark ring (not obtained experimentally) is drawn to indicate the position of the object.

The 8 lines/mm resolution sections are positioned at these four positions. As it can be seen in Fig. $6 \mathrm{~b}$ and Fig. 6c the resolution does not seem to depend on the position since the difference between the maximum and the closest minimum is the same for all four position, i.e. in the horizontal and vertical directions respectively. The influence of the radial position of the ring is investigated by probing the object plane at different crystal angles. This can be accomplished by angle tuning of the crystal. In our case, changing this variable will modify the ring radius and probe different parts of the object. In order always to illuminate the same part of the resolution target ( 8 lines $/ \mathrm{mm}$ ), the resolution target has to be radially translated for each selected crystal angle (see Fig. 7a).

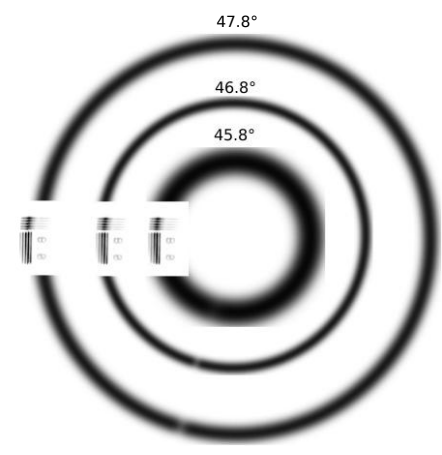

(a)

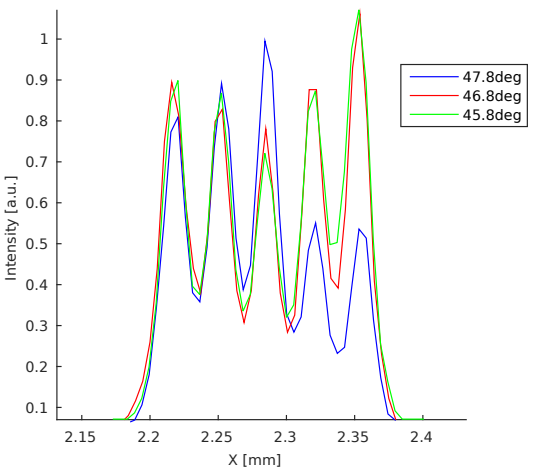

(b)

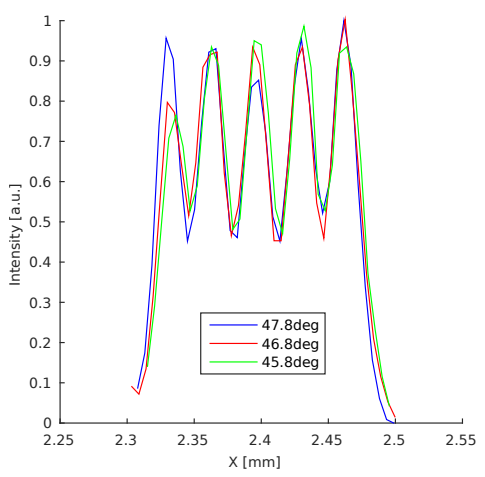

(c)

Figure 7: The 8 lines $/ \mathrm{mm}$ is probed at three different radial positions, each of them are produced from three different crystal angles. Linear intensity scan in the (b) radial and (c) tangential direction. The dark rings in (a) were not obtained experimentally but drawn to indicate the position of the object.

As can be observed from Fig. 7b and Fig. 7c, the resolution is not affected by the radial position of the ring, however the thickness of the ring depends on its radius. The ring produced experimentally (actual image not 
shown) from a crystal angle of $47.8^{\circ}$ is much thinner than the one produced from an angle of $45.8^{\circ}$. The larger the ring radius the thinner the ring.

\section{CONCLUSION}

In this paper we have investigated the upconversion imaging process using a mid-infrared light beam to illuminate the object. The origin of the ring shaped intensity distribution in the image plane is produced from multiple non-collinear phase matching solutions, the solutions being symmetrically located in angle with respect to the single phase matching solution.

An experimental setup has been built, using a 1.9 um signal beam and a 1.55 pump beam, both pulsed with a pulse width of $1 \mathrm{ps}$. The intensity and the spectrum both proved the symmetric nature of the phase matching solutions as well as the conservation of the bandwidth of the conversion process - also for a polychromatic source. A resolution target with 8 lines/mm was used to study the imaging resolution for a varying bandwidth of the signal beam as well as for different positions in the object plane. The results from this experimental work lead to the conclusion that neither the spectrum of the signal beam nor the position of the object plane affects the resolution of the upconverted image significantly.

\section{FUNDING}

Mid-TECH H2020-MSCA-ITN-2014 Grant agreement no: 642661

\section{REFERENCES}

[1] Kim, S.-S., Young, C., Vidakovic, B., and Mizaikoff, B., "Potential and challenges for mid-infrared sensors in breath diagnostics," IEEE Sens. J. 10, 145-158 (2010).

[2] Gurski, T. R., Epps, H. W., and Maran, S. P., "Astronomical demonstration of an infrared upconverter," Nature 249, 638 - 639 (1974).

[3] Imaki, M. and Kobayashi, T., "upconverter for high-sensitivity imaging of gas plumes," Opt. Lett. 32, 19231925 (2007).

[4] Huot, L., Moselund, P. M., Tidemand-Lichtenberg, P., Leick, L., and Pedersen, C., "Upconversion imaging using an all-fiber supercontinuum source," Opt. Lett. 41, 2466-2469 (2016).

[5] Burda, C., Link, S., Mohamed, M., and El-Sayed, M., "The relaxation pathways of cdse nanoparticles monitored with femtosecond time-resolution from the visible to the ir: assignment of the transient features by carrier quenching," J. Phys. Chem. B 105, 1228612292 (2001).

[6] Bonetti, C., Alexandre, M. T. A., van Stokkum, I. H. M., Hiller, R. G., Groot, M. L., van Grondellea, R., and Kennis, J. T. M., "Identification of excited-state energy transfer and relaxation pathways in the peridininchlorophyll complex: an ultrafast mid-infrared study," Phys. Chem. Chem. Phys. 12, 9256-9266 (2010).

[7] Nienhuys, H.-K., Woutersen, S., van Santen, R. A., and Bakker, H. J., "Mechanism for vibrational relaxation in water investigated by femtosecond infrared spectroscopy," J. Chem. Phys. 111, 1495-1500 (1999). 